\title{
Proteasome Inhibitors Prevent Caspase-1-Mediated Disease in Rodents Challenged with Anthrax Lethal Toxin
}

\author{
Stefan M. Muehlbauer, ${ }^{*}$ Heriberto Lima, Jr., ${ }^{*}$ \\ David L. Goldman, ${ }^{\dagger}$ Lee S. Jacobson, ${ }^{*}$ \\ Johanna Rivera, ${ }^{*}$ Michael F. Goldberg, ${ }^{*}$ \\ Michael A. Palladino, ${ }^{\ddagger}$ Arturo Casadevall, ${ }^{*}$ \\ and Jürgen Brojatsch* \\ From the Departments of Microbiology and Immunology, " and \\ Pediatrics and Infectious Disease, ${ }^{\dagger}$ Albert Einstein College of \\ Medicine, Bronx, New York; and Nereus Pharmaceuticals, ${ }^{\ddagger}$ \\ San Diego, California
}

NOD-like receptors (NLRs) and caspase-1 are critical components of innate immunity, yet their over-activation has been linked to a long list of microbial and inflammatory diseases, including anthrax. The Bacillus anthracis lethal toxin (LT) has been shown to activate the NLR Nalp1b and caspase-1 and to induce many symptoms of the anthrax disease in susceptible murine strains. In this study we tested whether it is possible to prevent LT-mediated disease by pharmacological inhibition of caspase-1. We found that caspase-1 and proteasome inhibitors blocked LT-mediated caspase-1 activation and cytolysis of LT-sensitive (Fischer and Brown-Norway) rat macrophages. The proteasome inhibitor NPI-0052 also prevented disease progression and death in susceptible Fischer rats and increased survival in BALB/c mice after LT challenge. In addition, NPI-0052 blocked rapid disease progression and death in susceptible Fischer rats and BALB/c mice challenged with LT. In contrast, Lewis rats, which harbor LT-resistant macrophages, showed no signs of caspase-1 activation after LT injection and did not exhibit rapid disease progression. Taken together, our findings indicate that caspase-1 activation is critical for rapid disease progression in rodents challenged with LT. Our studies indicate that pharmacological inhibition of NLR signaling and caspase- 1 can be used to treat inflammatory diseases. (Am J Pathol 2010, 177:735-743; DOI: 10.2353/ajpath.2010.090828)

Innate immunity plays a critical role in controlling microbial infections. Activation of the inflammasome, an integral part of the innate immune response, has been linked to cell death and morbidity triggered by Salmonella, Francisella, Listeria, and Staphylococcus. ${ }^{1-3}$ The inflammasome has also been linked to macrophage killing triggered by lethal toxin $(\mathrm{LT})$, a major virulence factor released by the Gram-positive bacterium Bacillus anthracis. ${ }^{4-8}$ In fact, when injected into small animals, LT alone is sufficient to reproduce the majority of the symptoms of the anthrax disease. Small molecule inhibitors of $L T$ have been shown to enhance survival of rodents challenged with $B$. anthracis., 9,10

The NOD-like receptor (NLR) Nalp1b controls LT susceptibility in murine macrophages. ${ }^{4,11} \mathrm{Nalp} 1 \mathrm{~b}$ is highly polymorphic in mice, and macrophages from strains expressing a dominant allele of Nalp1b are susceptible to rapid necrosis after LT exposure. ${ }^{4,12,13}$ Conversely, murine macrophages expressing a recessive Nalp $1 \mathrm{~b}$ allele undergo slow, caspase-1 independent apoptosis in response to LT challenge. ${ }^{6,13}$ We have shown that stimulation of the Nalp1b inflammasome by LT results in caspase- 1 activation in susceptible murine macrophages. ${ }^{6,14,15}$ Moreover, caspase- 1 activation in these cells is essential for LT killing. ${ }^{4,7,13,16}$ We also demonstrated that LT-mediated caspase- 1 activation and subsequent necrosis in susceptible murine macrophages are controlled by proteasome activity. ${ }^{5-7,17}$ In fact, proteasome inhibitors are the most efficient inhibitors of LT/caspase-1-mediated macrophage killing identified. ${ }^{5-7,17,18}$

Supported in part by the grant 5U54AI057158-05 from the Northeastern Biodefense Center (D.L.G. and A.C.), by the NIH Medical Scientist Training grant T32GM007288 (S.M.M.), and by a grant (NIAID-AI075222-01A1 to J.B.) from the National Institute of Allergy and Infectious Disease.

Accepted for publication April 1, 2010.

M.A.P. is an employee of Nereus Pharmaceuticals, Inc., which produces NPI-0052. None of the other authors declare any relevant financial relationships.

Supplemental material for this article can be found on http://ajp. amjpathol.org.

A guest editor acted as editor-in-chief for this article. No person at Thomas Jefferson University or Albert Einstein College of Medicine was involved in the peer review process or final disposition for this article.

Address reprint requests to Jürgen Brojatsch, Ph.D., Albert Einstein College of Medicine, Department of Microbiology and Immunology, Golding 404, 1300 Morris Park Ave, Bronx, NY 10461. E-mail: brojatsc@ aecom.yu.edu. 
As observed in mice, the susceptibility to $L T$ in rats is strain-dependent. Rats are divided into susceptible strains that are rapidly killed after LT challenge, and strains that are resistant to rapid disease progression. ${ }^{19}$ In contrast to mice, however, the in vivo LT susceptibility of rat strains closely mimics the in vitro susceptibility of their corresponding macrophages. ${ }^{19}$ Backcrossing experiments using susceptible and resistant rat strains suggest that the (in vivo and in vitro) LT susceptibility in rats is controlled by a single dominant gene. ${ }^{19}$ After LT administration, rats develop pulmonary edema, and die from a vascular collapse, a hallmark of human anthrax. ${ }^{20-22}$ Due to greater similarities with human anthrax, the rat model of LT-induced disease may be superior to the murine model.

We hypothesized that macrophage killing and cytokine release are critical events in the death of susceptible rats. Due to the correlation in rats between in vivo and in vitro LT susceptibility, we proposed that LT-mediated macrophage killing controls the rapid disease progression seen in susceptible rats. Due to the correlation between the murine and rat systems, we hypothesized that the single dominant gene controlling rat susceptibility is the rat homologue to murine Nalp1b. If true, caspase-1 activation would also control macrophage killing. We found that caspase- 1 and proteasome inhibitors prevented caspase-1 activation and macrophage cytolysis mediated by LT. Furthermore, the proteasome inhibitor NPI0052 prevented disease progression and mortality in LT-treated susceptible rats. Proteasome inhibitors also increased survival in BALB/c mice challenged with LT. Taken together, we demonstrated that caspase-1 activation not only controls macrophage killing, but also disease progression in susceptible rats challenged with LT. Our findings suggest that drugs controlling the inflammasome and caspase- 1 are potential therapeutics for inflammatory diseases mediated by microbial pathogens.

\section{Materials and Methods}

\section{Animals, Cell Culture, and Reagents}

Male Fischer (F344), Brown-Norway (BN), Lewis (LEW), and Wistar (WKY) rats (200 to $300 \mathrm{~g}$ ) were obtained from Taconic Farms (Hudson, NY). Female BALB/c mice (6 to 8 weeks old) were obtained from the National Cancer Institute (Bethesda, MD). Boc-D-CMK and MG132 were obtained from Calbiochem (San Diego, CA). Salinosporamide-A (NPI-0052) and bortezomib (Velcade) were generous gifts from Nereus Pharmaceuticals (San Diego, CA) and Millenium Pharmaceuticals (Cambridge, MA), respectively. Tumor necrosis factor- $\alpha$, Camptothecin, and cycloheximide were purchased from Biovision (Mountainview, CA). Endotoxin-free recombinant lethal factor (LF) and protective antigen (PA) were kindly provided by the Northeast Biodefense Protein Expression Core (Albany, NY).

\section{Generation of Rat Bone Marrow-Derived Macrophages}

Rat bone marrow-derived macrophages (BMMs) were generated as previously described. ${ }^{23,24}$ Bone marrow cells were flushed from femora and tibiae of F344, LEW, $\mathrm{BN}$, and WKY rats. Cells were differentiated into macrophages by incubation for 7 days in Dulbecco's modified Eagle's medium supplemented with 20\% conditioned medium from a confluent culture of L929 fibroblasts as a source of CSF-1. After removal of nonadherent cells, macrophages were recovered by washing plates with cold PBS containing $5 \mathrm{mmol} / \mathrm{L}$ EDTA. BMMs were uniformly positive for ED1 (AbD Serotec, Raleigh, NC) staining.

\section{Cell Death and Caspase Activation Assays}

Cell viability was analyzed by the water-soluble tetrazolium (WST) assay, as described previously. ${ }^{15}$ Terminal deoxynucleotidyl transferase-mediated dUTP nick-end labeling and propidium iodide exclusion assays were performed as previously described. ${ }^{25,26}$ For analysis of caspase-1 activation, we used a fluorescent caspase-1 activity assay, FLICA, from Immunochemistry Technologies (Bloomington, MN). The assay was performed in 96-well plates, with $8 \times 10^{4}$ cells/well. Cells were incubated with LT and/or inhibitors, and stained in complete media with FLICA reagent (FAM-YVAD-FMK) for 2 hours. Cells were washed five times in PBS containing 10\% fetal bovine serum, and fluorescence was measured on a Perkin Elmer Victor 3 multititer plate reader. For analysis of caspase-3 activation, we used a colorimetric caspase-3 assay as recommended by the manufacturer, R\&D Systems (Minneapolis, MN). Electron microscopy was performed as described previously. ${ }^{27}$

\section{Western Blotting}

Western blotting was performed as previously described. ${ }^{6}$ Membranes were probed with a polyclonal antibody to MEK-3 (Santa Cruz Biotechnology, Santa Cruz, CA; number Sc-960), monoclonal antibody to actin (Ac40: Sigma, St. Louis, MO), and polyclonal antibody to rat interleukin (IL)-18 (MAB521; R\&D Systems) at dilutions of 1:500. Polyclonal horseradish peroxidase-conjugated antibodies to rat and mouse immunoglobulins (Sc-2313 and 2314; Santa Cruz Biotechnology) were used as the secondary antibodies, and blots were developed by using Luminol Enhancer Substrate (Fisher Scientific, Pittsburgh, PA).

\section{In Vivo Experiments}

Rats were injected intravenously with $150 \mu \mathrm{g} / \mathrm{kg}$ of LT or PBS. LT was prepared by diluting PA and LF in sterile PBS in a 4:1 ratio (PA:LF). Alternately, rats were injected with PBS only. Bortezomib and NPI-0052 were injected intravenously 60 minutes before LT injections. Bortezomib was dosed at $1 \mathrm{mg} / \mathrm{kg}$, and infused in a solution of 0.35 
$\mathrm{mg} / \mathrm{ml}$ in physiological saline. NPI-0052 was dosed at 0.2 $\mathrm{mg} / \mathrm{kg}$ and infused in a solution of $0.1 \mathrm{mg} / \mathrm{ml}$ in $5 \%$ Solutol (BASF Aktiengesellschaft, Germany). The final NPI-0052 solution contained $1 \%$ DMSO. In experiments using NPI0052, control animals and rats treated with LT-only received injections of the NPI-0052 vehicle solution 60 minutes before LT injection. Blood collections were made by puncture of the inferior vena cava. In rat survival assays, animals were monitored continuously for up to 4 hours post-LT injection. Surviving rats were sacrificed at 4 hours post-LT or PBS injection. Histopathological studies were performed as previously described. ${ }^{28}$

\section{Cytokine Multiplex Assay}

Rats were IV-injected with LT, NPI-0052, or both, and blood was collected as described above. Blood was kept on ice for 30 minutes to allow clotting, and then centrifuged at $4^{\circ} \mathrm{C}$ to separate the serum fraction. Sera were stored at $-80^{\circ} \mathrm{C}$, and submitted to the Albert Einstein College of Medicine Hormone Assay Core facility. The multiplex assay (RCYTO-80K-PMX24) was performed according to the manufacturer's instructions (Millipore, Billerica, MA).

\section{Analysis of Splenic Macrophages}

Rat spleens were harvested immediately after sacrifice, and splenocytes were obtained via mechanical disruption and passage through a 70- $\mu \mathrm{m}$ cell strainer. Red blood cells were eliminated with RBC lysis buffer (Sigma). For each staining condition, $10^{6}$ cells were resuspended in PBS and stained with the violet LIVE/DEAD viability dye (Molecular Probes, Eugene, OR) according to the manufacturer's protocol. Cells were washed and resuspended in flow cytometry buffer (PBS, $2 \%$ fetal bovine serum, $0.05 \% \mathrm{NaN}_{3}$ ). FC receptors were blocked with anti-rat CD32 (clone D34-485; BD Pharmingen, Carlsbad, CA). Surface markers were stained with Alexa Fluor 488-conjugated anti-CD3 and phosphatidylethanolamine-conjugated anti-CD11b/c (clones 1F4 and OX-42; Biolegend, San Diego, CA). After staining, cells were fixed with $4 \%$ paraformaldehyde in PBS, permeabilized with flow cytometry buffer containing $0.1 \%$ saponin, and stained with Alexa Fluor 647-conjugated anti-CD68 (clone ED1; $\mathrm{AbD}$ Serotec, Raleigh, NC). Cells were acquired on an LSRII flow cytometer (BD Biosciences, Palo Alto, CA), and analyzed by using FlowJo software (Tree Star, Ashland, OR).

\section{Statistical Methods}

Multiple comparisons of non-normally distributed data were performed by using the Kruskal-Wallis test followed by the Dunn's multiple comparison test. For normally distributed data, comparisons were performed by using analysis of variance followed by the Student-NewmanKeuls test. Survival differences were calculated by using the Kaplan-Meier statistic. Statistics were performed with
GraphPad Software (La Jolla, CA). $P$ values $<0.05$ were considered significant.

\section{Results}

LT killing of murine macrophages is strain-specific and dependent on activation of the inflammatory proteins Nalp 1b and caspase-1. ${ }^{4,29-31}$ Recent studies have indicated that LT susceptibility of rat macrophages is also strain-dependent and controlled by a single, dominant gene, potentially the rat homologue of murine Nalp $1 b .{ }^{19}$ Based on the similar LT susceptibility pattern of murine and rat macrophages, we predicted that LT killing of susceptible rat macrophages was also caspase-1-dependent. Genetic backcrossing experiments in rats further suggested that a dominant gene not only controlled LT killing in vitro, but also LT-mediated disease progression. ${ }^{19}$ This in vitro and in vivo correlation in rats is clearly distinct from the murine system, where in vitro and in vivo LT susceptibilities do not correlate. ${ }^{30,31}$ Based on these studies, we hypothesized that a dominant gene controls macrophage killing as well as vascular collapse in rats challenged with LT. To test this hypothesis we initially determined whether the inflammatory proteincaspase-1 controlled macrophage killing of susceptible rat macrophages. Based on the in vitro and in vivo correlation in rats, we further investigated whether inhibition of caspase- 1 could prevent macrophage killing and disease in LT-treated rats.

\section{LT Triggers Caspase-1-Mediated Cytolysis in F344 Rat Macrophages}

To determine whether LT activates the inflammatory caspase-1 in susceptible rat macrophages, we generated primary BMMs from susceptible and resistant rat strains. BMMs responded to a cytotoxic dose of LT (250 and $500 \mathrm{ng} / \mathrm{ml}$ of LF and PA, respectively $)^{27}$ in a straindependent fashion. F344 and BN-derived macrophages were rapidly killed within 2 to 4 hours by LT, whereas LEW and WKY BMMs were resistant to rapid LT-induced cytolysis (Figure 1A, and Supplemental Figure 1, C and D, at $h$ ttp://ajp.amjpathol.org).

Next we tested whether LT killing of susceptible rat macrophages was caspase-1-dependent. The caspase-1 inhibitor Boc-D-CMK blocked caspase-1 activation and cytolysis of LT-treated F344 macrophages (Figure 1, B and C) indicating that caspase-1 activation was required for LT killing of susceptible rat BMMs. Consistent with caspase-1dependence, ${ }^{6,12}$ we detected significant processing of the caspase- 1 substrates IL-18 and IL-1 $\beta$ in the lysates of F344 BMMs after LT exposure (Figure $1 D$ and Supplemental Figure 1B at $h$ ttp://ajp.amjpathol.org). Caspase-1 activation in LT-treated F344 BMMs was further confirmed by using the fluorogenic activated caspase-1 specific stain, FLICA (Figure 1C). As caspase-1-mediated cell death has been linked to necrosis induction, ${ }^{5,32-35}$ we tested for necrotic markers in LT-treated F344 macrophages. These showed several morphological features consistent with necrosis, in- 
A

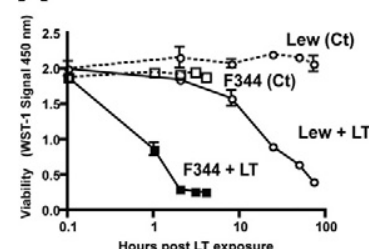

C

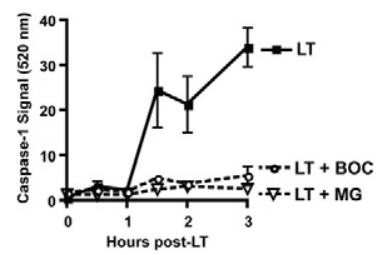

E

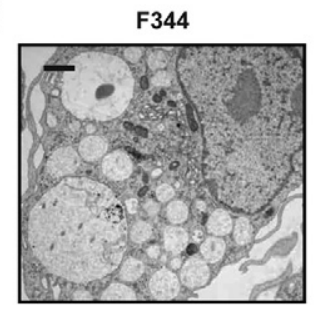

Untreated
B

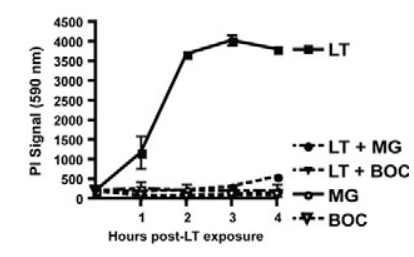

D

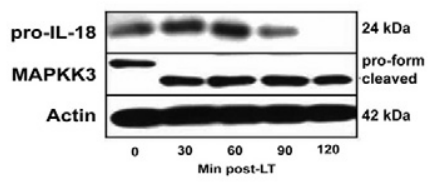

F344

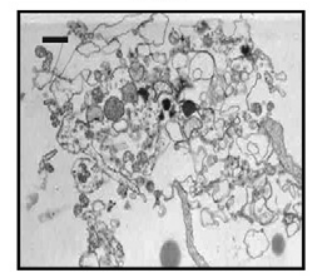

LT-treated

Figure 1. Caspase-1 activation is essential for LT-mediated necrosis in susceptible rat macrophages. A: F344 and LEW BMMs were subjected to LT (LF and PA: 250 and $500 \mathrm{ng} / \mathrm{ml}$ ), and cell viability was determined by WST-1 assay. B and C: F344 BMMs were treated with LT in the presence or absence of $80 \mu \mathrm{mol} / \mathrm{L}$ Boc-D-CMK or $10 \mu \mathrm{mol} / \mathrm{L}$ MG132. Membrane integrity was measured by analysis of propidium uptake $(\mathbf{B})$, and caspase-1 activation $(\mathbf{C})$ was determined by FLICA assay as indicated in arbitrary units. D: LT triggers processing of IL-18 in F344 BMMs after LT exposure. Cleavage of pro-IL-18 was determined by Western blotting of lysates from these cells, and actin is shown as a loading control. Representative data are shown from at least three independent experiments. Data are represented as mean $\pm \mathrm{SD}$ of $N=3$. E: LT-treated F344 BMMs show signs of necrotic cell death, as analyzed by electron microscopy 4 hours post-LT exposure (Scale bars $=1 \mu \mathrm{m}$ ).

cluding membrane impairment (as measured by propidium iodide uptake; Figure 1B), loss of organelle, and plasma membrane integrity (as measured by electron microscopy; Figure 1E).

Although LEW macrophages were resistant to rapid cytolysis after LT exposure, these cells still died 48 to 72 hours post-LT exposure and showed a similar LT-dose response as F344 macrophages (Figures 1A and 2A). Similar results were obtained with WKY macrophages (Supplemental Figure 1C at http://ajp.amjpathol.org). Although LT did not activate caspase-1 in LEW BMMs, it

triggered caspase-3 activation and a terminal deoxynucleotidyl transferase-mediated dUTP nick-end labeling signal consistent with apoptosis induction in these cells (Supplemental Figure 1E at http://ajp.amjpathol.org). Of note, we found that caspase-3 was not activated in LTtreated in F344 BMMs (Figure 2, B and C). Collectively our findings indicate that LT triggers caspase-1-mediated necrosis, also termed pyroptosis, ${ }^{5}$ in "susceptible" F344 rat macrophages, and slow cell death in LEW and WKY macrophages.

\section{Proteasome Inhibitors Block LT Killing of Susceptible Rat Macrophages by Preventing Caspase-1 Activation}

We have provided evidence that the LT susceptibility of F344 (and BN) macrophages is controlled by the inflammatory protein caspase-1. As the LT susceptibilities of rat macrophages to LT correlates to in vivo susceptibilities, we then tested whether blocking caspase- 1 not only prevents LT killing of susceptible rat macrophages, but also prevents disease progression in susceptible rats challenged with LT. To determine the contribution of caspase-1 activation to disease progression in vivo, we set out to block caspase-1 activation in susceptible F344 rats challenged with LT. Caspase- 1 inhibitors, however, are poor choices to block caspase-1 activation in vivo, due to their poor solubility and high cytotoxicity. As an alternative approach, we focused on proteasome inhibitors, which efficiently block LT killing of murine macrophages, ${ }^{7,15,36}$ and which have been used in vivo. ${ }^{37-40}$

Consistent with findings in murine macrophages, we found that the proteasome inhibitor MG132 blocked LT killing of susceptible rat (F344) BMMs (Figure 1, B and C). The water-insoluble MG132, however, is like the general caspase-1 inhibitor Boc-d-cmk, not suitable for in vivo usage. We therefore focused on the water-soluble proteasome inhibitors bortezomib and salinosporamide-A (NPI-0052), which have been widely used in animals and humans. ${ }^{37-40}$ Bortezomib and NPI-0052 effectively blocked cytolysis in primary F344 BMMs at significantly lower concentrations than MG132 (Figure 3A). NPI-0052 was particularly potent, protecting macrophages at concentrations 100 times lower than MG132 and approximately 20 times lower than bortezomib (Figure $3 \mathrm{~A}$ ). These findings suggest that proteasome inhibi-

A

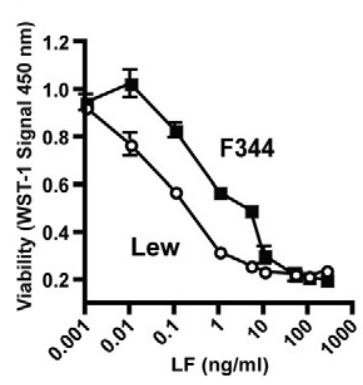

B

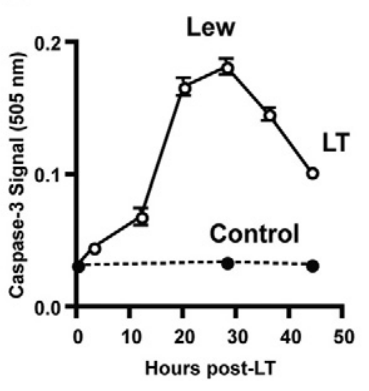

C

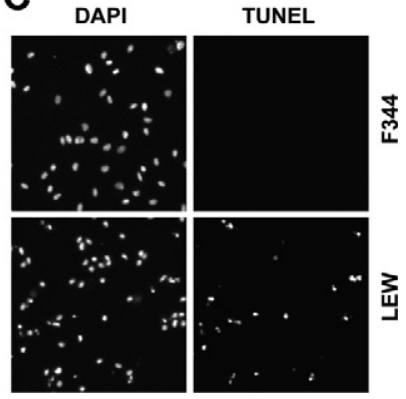

Figure 2. Strain-specific killing of rat macrophages. A: F344 and LEW BMMs are equally susceptible to LT killing. F344 and LEW BMMs were subjected to PA $(500 \mathrm{ng} / \mathrm{ml})$ and varying concentrations of LF. Cell viability of F344 and LEW BMMs was determined by WST-1 assay 2 and 48 hours post LT exposure, respectively. B: LT triggers caspase- 3 in LEW macrophages, as determined by a colorimetric caspase- 3 cleavage assay. C: F344 and LEW BMMs were exposed to LT and subjected to DAPI and terminal deoxynucleotidyl transferase-mediated dUTP nickend labeling staining 6 and 72 hours post-LT exposure, respectively. Representative data are shown from at least three independent experiments. Data are represented as mean $\pm \mathrm{SD}$ of $N=3$. 

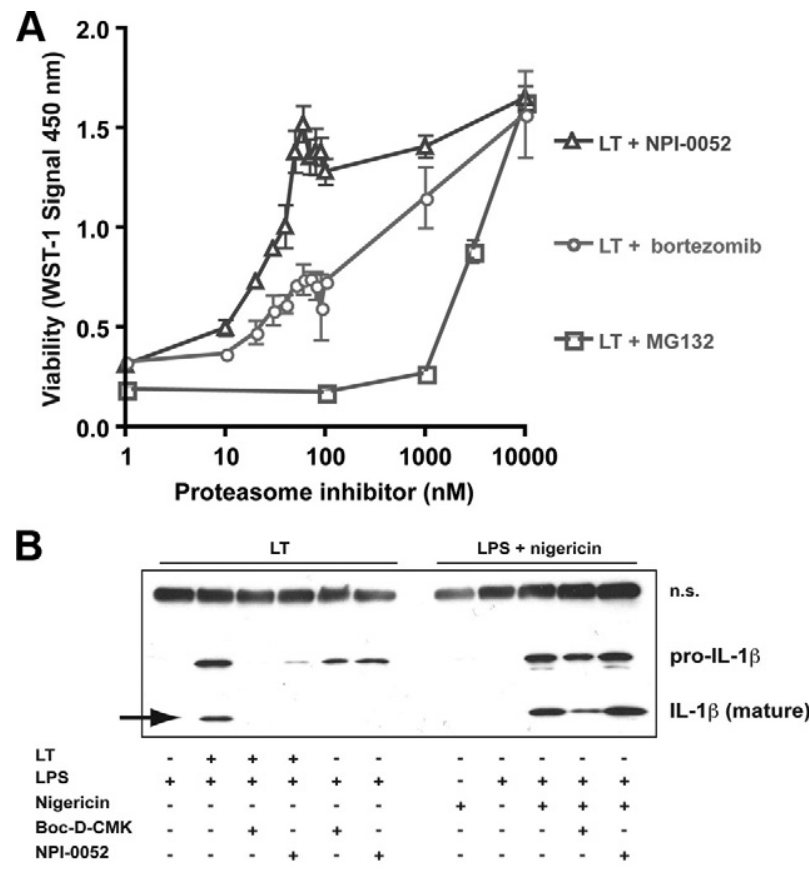

C

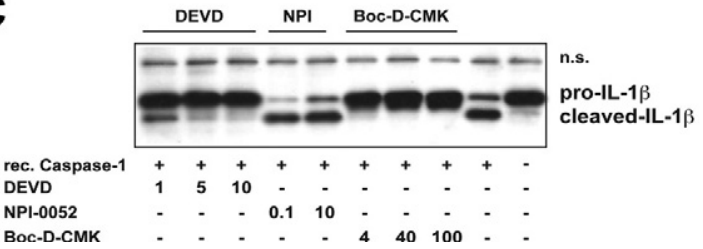

Figure 3. Proteasome inhibitors block caspase-1-mediated LT killing of F344 macrophages. A: The proteasome inhibitors MG132, NPI-0052, and bortezomib were applied at the given concentrations to F344 BMMs treated with LT. Viability was established by WST-1 assay 4 hours after LT-treatment. Data are represented as mean $\pm \mathrm{SD}$ of $N=3$, and representative data are shown from three independent experiments. B: The proteasome inhibitor NPI-0052 does not block caspase-1 directly. Lysates from LPS-primed J344 BMMs were incubated with recombinant caspase- 1 in the presence of NPI0052 and Boc-d-cmk for 2 hours. Western blots were performed with an IL-1 $\beta$ antibody to monitor caspase-1 activity. N.s. indicates nonspecific bands. Arrow points to mature IL-1 $\beta$. C: NPI-0052 did not prevent caspase-1 activation and cytolysis of F344 BMMs mediated by the Nalp3-inducers LPS and nigericin. F344 BMMs were incubated with LPS and nigericin in the presence of NPI-0052 (uM) and Boc-d-cmk (uM) for 4 hours. IL-1 $\beta$ processing was measured by Western blotting and cell survival was determined by LDH release.

tors are efficient inhibitors of LT killing of F344 macrophages.

We next tested whether the proteasome inhibitor NPI0052 blocked LT killing of F344 BMMs by directly targeting caspase-1. As suggested for MG132, ${ }^{7,15} \mathrm{NPI}-0052$ (and the caspase-1 inhibitor Boc-d-cmk) efficiently prevented processing and release of mature $\mathrm{IL}-1 \beta$ in LTtreated F344 macrophages (Figure 3B). However, NPI0052 did not prevent caspase-1 activation and cytolysis of F344 BMMs mediated by the Nalp3-inducers LPS and nigericin (Figure $3 \mathrm{~B}$ ). Using an in vitro $\mathrm{IL}-1 \beta$ cleavage assay with recombinant caspase-1, we found that NPI0052 does not target caspase-1 directly (Figure 3C). $\mathrm{NPI}-0052$ did not prevent IL-1 $\beta$ processing by recombinant caspase-1 in lysates from LPS-treated F344 BMMs (Figure 3C). As positive controls, we used the caspase-1 inhibitor Boc-d-cmk and the pan caspase inhibitor zVAD-FMK, both of which blocked IL-1 $\beta$ processing (Fig-
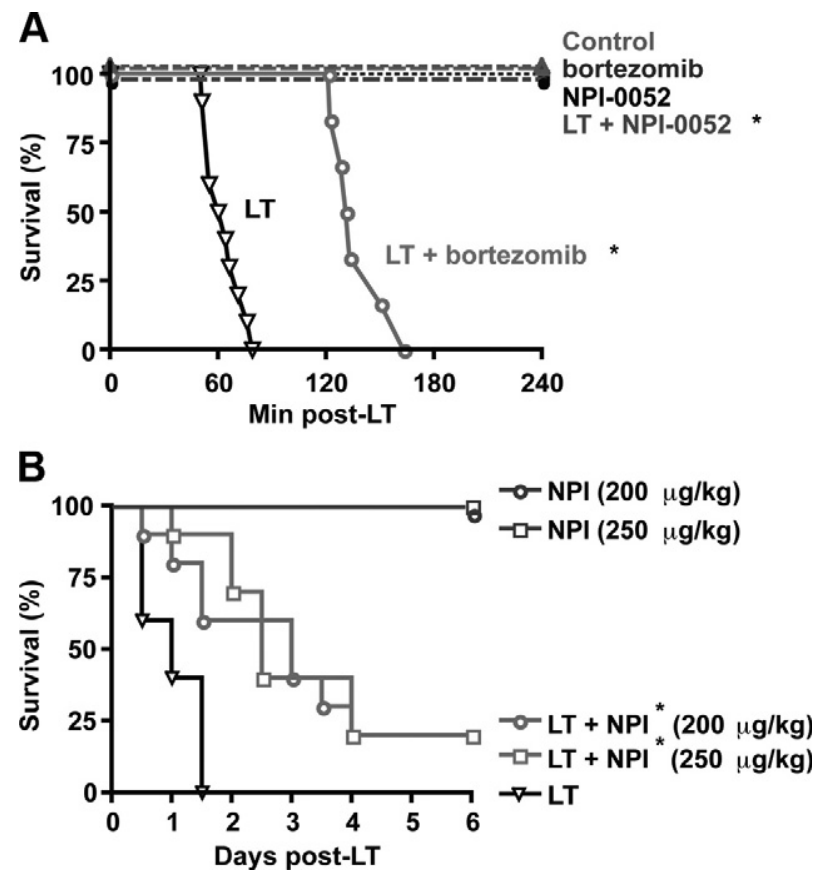

Figure 4. Proteasome inhibitors block LT killing in susceptible rat and murine strains. A: F344 rats (250 to $300 \mathrm{~g}$ ) were injected IV with $1.0 \mathrm{mg} / \mathrm{kg}$ bortezomib (bortezomib only: $N=6$ ) or $0.2 \mathrm{mg} / \mathrm{kg}$ NPI-0052 plus vehicle (NPI-0052 only: $N=6$ ), followed by IV injection of either PBS or $0.15 \mathrm{mg} / \mathrm{kg}$ LT. Rat survival up to 240 minutes was measured following PBS (control: $N=$ 5) or LT (LT only: $N=10$, mean survival times: 59.7 minutes; LT + bortezomib, mean survival times: 135.4 minutes: $N=6$; LT $+0052: N=9$ ) injections. Representative data are shown from three independent experiments. ${ }^{*} P<0.05$ for comparison with LT treatment alone. B: BALB/c mice were injected IV with NPI-0052 plus vehicle ( $200 \mu \mathrm{g} / \mathrm{kg}$ NPI-0052 only: $N=$ 6; $250 \mu \mathrm{g} / \mathrm{kg}$ NPI-0052 only: $N=6$ ), followed by IV injection of either PBS or $0.15 \mathrm{mg} / \mathrm{kg}$ LT $(\mathrm{LT}+200 \mu \mathrm{g} / \mathrm{kg} \mathrm{NPI-0052:} N=10 ; \mathrm{LT}+250 \mu \mathrm{g} / \mathrm{kg}$ NPI-0052 only: $N=10$ ). Survival up to six days was measured. Representative data are shown from three independent experiments. ${ }^{*} P<0.05$ for comparison with LT treatment alone.

ure 3C). Taken together our data suggest that the proteasome inhibitor NPI-0052 does not directly block caspase- 1 activation in F344 BMMs, but likely acts upstream in the formation or activation of the LT-induced imflammasome complex.

\section{Proteasome Inhibitors Block LT-Induced Disease Progression in Susceptible Rats}

We have demonstrated that proteasome inhibitors efficiently prevent caspase- 1 activation and cytolysis of susceptible rat macrophages. We next wanted to know whether the water-soluble proteasome inhibitors bortezomib and NPI-0052 could also prevent caspase-1 activation and disease progression in LT-treated F344 rats. To evaluate the effects of proteasome inhibitors on LTinduced disease, we injected F344 rats intravenously with bortezomib or NPI-0052 60 minutes before challenge with a lethal dose of LT. All (100\%) of the control F344 rats challenged with LT died within 80 minutes of LT challenge, with a median survival time of 60 minutes (Figure $4 A$ ). F344 rats challenged with LT in the presence of 1 $\mathrm{mg} / \mathrm{kg}$ of bortezomib, however, survived more than twice as long, with a median survival time of 131 minutes (Figure 4A). Strikingly, F344 rats treated with NPI-0052 plus 
LT remained alive throughout the duration of experiment, with $100 \%$ survival at 240 minutes after LT challenge (Figure 4A). Our finding that NPI-0052 was more efficient in blocking death in vivo than bortezomib was consistent with our in vitro data, showing that NPI-0052 was more efficient than bortezomib in blocking necrosis of F344 macrophages (Figure 3A).

We were unable to perform long-term studies with $\mathrm{NPI}-0052$ in rats, as the proteasome inhibitor induced toxic effects in rats after 300 minutes (data not shown). Mice, however, show significantly fewer side effects than rats following NPI-0052 challenge (S. Neuteboom, personal communication). We therefore challenged LT-susceptible BALB/c mice with $L T$ in the presence and absence of NPI-0052. NPI-0052 showed no toxicity in the inhibitor-only group for the duration of the assays (6 days; Figure 4B). NPI-0052 shifted the survival curve of LT-treated BALB/C-treated macrophages by approximately 48 hours (Figure 4B). NPI-0052 treatment of LT-treated BALB/c mice increased survival by approximately $20 \%$, whereas no animal survived in the LT only group (Figure 4B).

To analyze the apparent rescue by proteasome inhibitors, we examined several key parameters associated with vascular collapse, such as pleural effusions, pulmonary edema, and hemoconcentration in F344 rats challenged with LT in the presence of NPI-0052. LT challenge alone caused massive hemoconcentration in F344 rats, with an average hematocrit of $80 \% 70$ minutes post-LT injection, compared with the $50 \%$ hematocrit measured in untreated (PBS) animals (Figure 5A). NPI-0052 prevented the LT-mediated increase in hematocrit in F344 rats (Figure 5A). Rats that received either vehicle only (data not shown), NPI-0052 alone, or NPI-0052 in combination with LT showed hematocrit levels consistent with those found in control animals (Figure 5A). Significant pleural effusions $(1.5 \mathrm{ml})$ were collected from the pleural cavities of F344 rats challenged with $L T$, whereas less than $0.01 \mathrm{ml}$ fluid was recovered from untreated control or (NPI$0052 \pm$ LT) treated rats (Figure 5B). In addition, we observed significantly increased lung weights in LTtreated F344 rats, consistent with severe pulmonary edema (Figure 5C). Left lungs were over $1.7 \mathrm{~g}$ in LTtreated rats, compared with $0.4 \mathrm{~g}$ in untreated rats (Figure $5 \mathrm{C}$ ). Massive and widespread pulmonary edema in the lungs of LT-treated rats was confirmed by histology (Figure 5D). Lung weights from F344 rats challenged with $\mathrm{LT}$ in the presence of NPI-0052, however, remained on par with control animals, and histological examination revealed no abnormalities, confirming the absence of pulmonary edema in rats challenged with LT and NPI0052 (Figure 5, C and D). As expected, no increase in hematocrit, no pleural effusion, and no changes in lung weight were observed in LEW rats up to 12 hours post-LT exposure (data not shown). Taken together, our data indicated that proteasome inhibitors block all signs of cardiovascular failure in LT-treated F344 rats.

\section{LT Induces Caspase-1 Activation and Macrophage Depletion in F344 Rats}

As LT killing of F344 macrophages is caspase-1-dependent, we tested whether caspase- 1 activation and macrophage depletion also occurred in vivo in rats challenged with LT. As an indicator of caspase- 1 activation in vivo, we measured levels of the caspase- 1 substrate IL-18 in the serum of LT-treated rats by using a Luminexbased cytokine multiplex assay. ${ }^{41}$ Of the cytokines tested, only IL-18 was increased in the sera of LT-treated F344 rats (Figure 6A). The levels of IL-18 in sera from F344 rats increased with higher LT doses. NPI-0052 treatment prevented the IL-18 burst observed in F344 rats (Figure $6 \mathrm{~B}$ ), consistent with the ability of proteasome inhibitor to prevent caspase-1 activation in LTtreated macrophages (Figures $1 \mathrm{C}$ and $3 \mathrm{~B}$ ). No IL-18 burst was measured in resistant LEW rats (data not shown), consistent with a lack of caspase-1 activation in LT-treated LEW macrophages (Supplemental Figure $1 \mathrm{~A}$ at $h$ ttp://ajp.amjpathol.org), and with the failure of
A

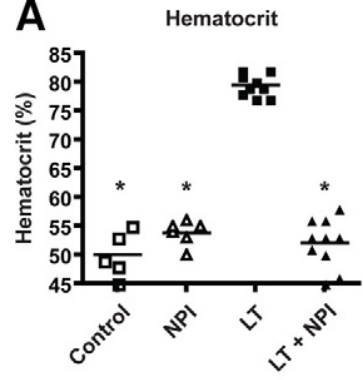

D

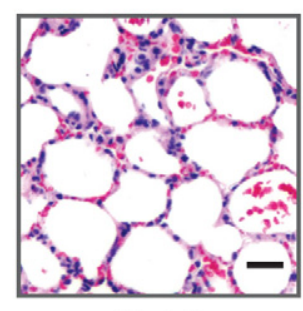

Control
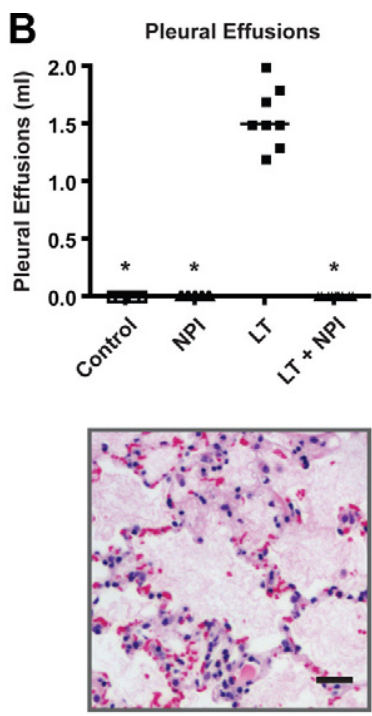

LT
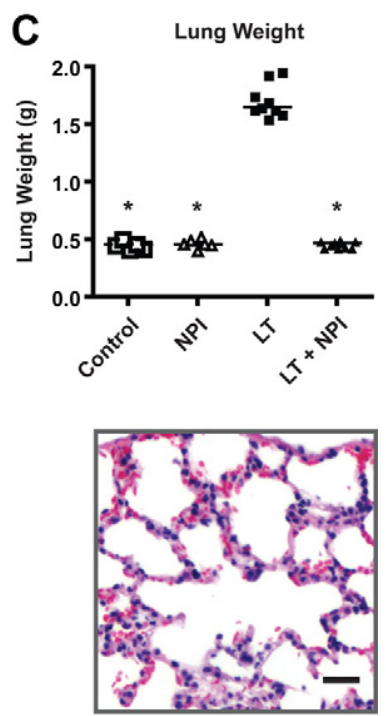

LT + NPI
Figure 5. NPI-0052 treatment prevents vascular defects induced by LT in F344 rats. A-C: F344 rats (250 to $300 \mathrm{~g}$ ) were either mock injected with vehicle and PBS (control: $N=5$ ), vehicle and LT $(N=9)$, NPI-0052 $(N=6)$ and PBS, or NPI-0052 and LT $(N=10)$. Moribund animals, present only in the LT only group, were sacrificed on loss of righting reflex; surviving animals were sacrificed four hours post injection. Blood was collected, and hematocrit measured (A). Clear pleural effusions were drained and quantified from opened thoracic cavities (B). Left lungs were removed, blotted on tissue paper, and weighed (C). D: Left lungs of untreated (PBS), LT-treated and LT/NPI-0052-treated rats were then preserved and analyzed for pulmonary edema by microscopy. Representative data are shown from at least three independent experiments. Scale bars $=100 \mu \mathrm{m} .{ }^{*} P<0.05$ for comparison with LT treatment alone. 
A

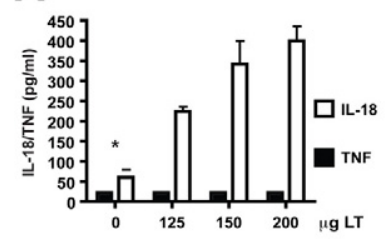

B

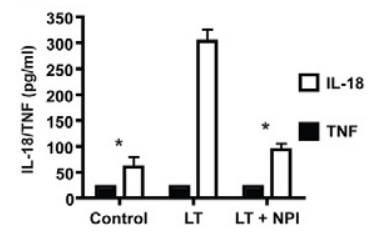

C
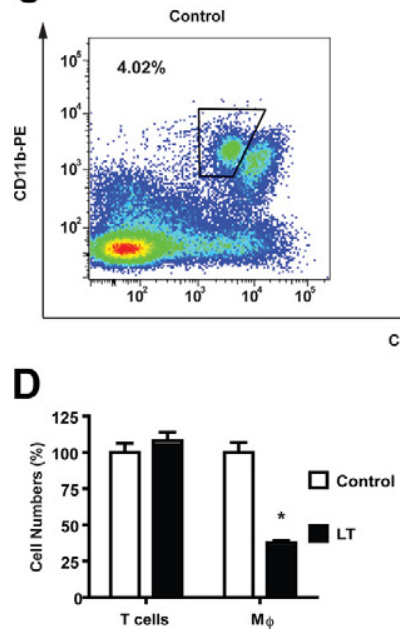

CD63-APC

E

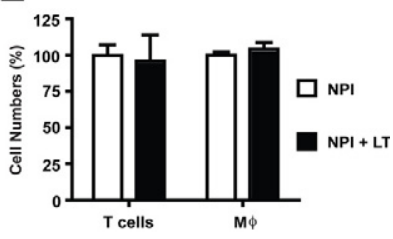

Figure 6. Macrophage depletion in LT-treated F344 rats. A-E: F344 rats (250 to $300 \mathrm{~g}$ ) were either injected with vehicle and PBS, vehicle and LT, NPI-0052 and PBS, or NPI-0052 and LT. Blood serum (A and B) and spleens $(\mathbf{C}-\mathbf{E})$ were collected 80 minutes post-LT injection. $\mathbf{A}$ and $\mathbf{B}$ : Levels of tumor necrosis factor and IL-18 were determined by Luminex-based multiplex assay. Representative data are shown from at least two independent experiments and are represented as \pm SD of $N=3 .{ }^{*} P<0.05$ for comparison with LT treatment alone. C: Representative flow cytometry plots of untreated and LT-treated F344 rats are shown and gates are drawn around live splenic macrophages $\left(\mathrm{CD} 3^{-}, \mathrm{ED} 1^{\text {int }}, \mathrm{CD} 11 \mathrm{~b} / \mathrm{c}^{+}\right)$. $\mathbf{D}$ and $\mathbf{E}$ : The percentage of macrophages (ED ${ }^{+}$, $\left.\mathrm{CD} 11 \mathrm{~B} / \mathrm{C}^{+}\right)$and $\mathrm{T}$ cells $\left(\mathrm{CD}^{+}\right)$were measured relative to controls. Macrophage and $\mathrm{T}$ cell populations were determined in untreated and LT-treated F344 rats (D), as well as in NPI-0052 and LT/NPI-0052-treated F344 rats (E). Representative data are shown from two independent experiments and are represented as $\pm \mathrm{SD}$ of $N=3 .{ }^{*} P=0.001$ for comparison with LT treatment.

resistant murine strains to mount a cytokine storm in response to $L T .21,31$

To assess the effect of LT on macrophage levels in vivo, we analyzed the splenic macrophage population in F344 rats treated with LT. We observed a greater than twofold reduction in splenic macrophages in LT-treated F344 rats, consistent with macrophage lysis in vivo (Figure $6, C$ and D). Although the splenic macrophage population was reduced after LT injections, levels of LTresistant cell populations (eg, T cell lymphocytes) did not change on LT exposure (Figure 6D). Consistent with our findings demonstrating that proteasome inhibitors blocked LT killing of macrophages in vitro, NPI-0052 treatment also prevented macrophage depletion in LTtreated F344 rats. As predicted, macrophage counts did not drop in rats challenged with LT and NPI-0052, compared with those treated with LT only, indicating that NPI-0052 prevents LT-mediated macrophage killing in vivo (Figure 6E).

Taken together, our findings indicate that LT triggers caspase-1-mediated necrosis in susceptible rat macrophages. We demonstrated that proteasome inhibitors prevent LT-mediated killing of F344 macrophages, pre- sumably by blocking an upstream event in caspase-1 activation. Proteasome inhibitors not only prevented macrophage killing by $L T$, but also significantly delayed LTinduced vascular collapse in susceptible mice and rats. These findings are consistent with the hypothesis that the pro-inflammatory protein caspase-1 contributes to rapid disease progression in LT-treated rodents, and that it is possible to pharmacologically control inflammatory diseases by targeting caspase-1 activation.

\section{Discussion}

\section{LT Triggers Caspase-1-Mediated Necrosis in Susceptible Rat Macrophages}

In mice, genetic backcrossing experiments have mapped the LT susceptibility locus to a dominant gene, the NOD-like receptor Nalp1b. ${ }^{4,29}$ Activation of Nalp1b results in caspase-1-mediated necrosis (pyroptosis) of murine macrophages. ${ }^{5-7,11,14,15}$ Nalp1b-mediated pyroptosis of murine macrophages is specifically blocked by caspase- 1 and proteasome inhibitors. ${ }^{5,7,15}$ Here we present evidence that LT also triggers caspase-1-mediated necrosis in susceptible rat macrophages. We describe that LT-treated susceptible rat macrophages also show signs of necrosis, and that proteasome inhibitors are able to block LT killing of rat macrophages. It has been previously demonstrated that a dominant gene controls LT killing of rat macrophages. ${ }^{19}$ NLRs tightly control caspase- 1 activation, and are the only known activators of caspase-1. Thus, based on the similarities between murine and rat macrophages, it is reasonable to assume that the rat homologue of the murine Nalp1b and caspase-1 control LT killing of rat macrophages.

\section{Proteasome Inhibitors Block an Upstream Step of LT-Mediated Caspase-1 Activation}

We have demonstrated that proteasome inhibitors block LT-mediated caspase-1 activation and necrosis of rat macrophages. Here we provide evidence that a novel proteasome inhibitor, NPI-0052, does not target caspase-1 directly, as the inhibitor fails to block recombinant caspase-1 and caspase-1 activation by Nalp3 inducers. ${ }^{5,15}$ As proteasome inhibitors do not interfere with LT uptake, ${ }^{7,15,36}$ our findings suggest that the proteasome inhibitor NPI-0052 blocks a step upstream of caspase- 1 activation, possibly by degrading a protective factor that inhibits activation of the Nalp1b inflammasome. This process appears to be NLR-specific, as these inhibitors prevent caspase- 1 activation by LT (presumably Nalp1b), but not by Nalp3 inducers.

$\mathrm{NPI}-0052$ is a new therapeutic proteasome inhibitor by Nereus Pharmaceuticals, and currently in stage 2 of clinical trials. It is bicyclic $\beta$-lactone $\gamma$-lactam and thus differs significantly from peptide-based inhibitors currently available. The dipeptide boronic-acid-based inhibitor bortezomib, also used in this study, binds to the 26S-proteasome, and is highly effective in multidrug resistant 
cancers. NPI-0052 is a more potent inhibitor than bortezomib and also exhibits fewer side effects. Both inhibitors have similar $I_{50 \mathrm{~s}}(5$ to $10 \mathrm{nmol} / \mathrm{L}$ ) in vivo (Nereus Pharmaceuticals).

\section{Proteasome Inhibitors Delay Disease Progression in LT-Treated Rodents}

As the single dominant gene that controls $L T$ killing of rat macrophages also control LT susceptibility of rats in vivo, we hypothesized that the same factor controlled both processes. Thus, we examined whether caspase- 1 activation also drives disease progression in rats challenged with LT. We found that the proteasome inhibitor NPI-0052 not only prevented LT killing of F344 macrophages but also increased survival of F344 rats after LT challenge. Susceptibility of mice to LT-mediated disease is much less tightly correlated to LT-induced cell death. However, a single bolus NPI-0052 treatment resulted in significant protection to susceptible (BALB/c) mice. NPI-0052 rescued $20 \%$ of LT-treated BALB/C mice, and extended survival of the mice by at least 2 days. The survival of $\mathrm{BALB} / \mathrm{c}$ mice challenged with $\mathrm{LT}$ and NPI-0052 was similar to that obtained with "resistant" (C57BL/6) mice, which lack the susceptible Nalp1b allele and do not undergo caspase-1-mediated necrosis in response to $\mathrm{LT}^{6}{ }^{6}$ It is possible that continuous dosing of NPI-0052, instead of a single bolus, would in fact result in further protection of LT-challenged mice.

It remains to be shown how caspase-1 activation contributes to LT-mediated disease progression in rats and mice. LT treatment of susceptible mice is known to result in a significant increase in serum levels of the caspase-1 substrates IL-1 $\beta$ and IL $18 .{ }^{30,31}$ In fact, the cytokines are part of a broad cytokine storm in mice harboring susceptible macrophages, but not in those with resistant macrophages. ${ }^{30,31}$ As only a fraction of caspase- 1 is generally activated under optimal conditions (in vitro) on stimulation of Nod-like receptors, we tested levels of the caspase- 1 substrate in the serum of LT-treated rats. We detected increased IL-18 levels in susceptible, but not in resistant rats after $\mathrm{LT}$ exposure. Moreover, the proteasome inhibitor NPI-0052 prevented an increase in IL-18 levels and a drop in macrophage counts in LT-treated F344 rats. Increased IL-18 levels in the serum and macrophage depletion is consistent with caspase- 1 activation in LT-treated F344 rats. Collectively, our findings suggest that activation of the inflammatory response, in particular of caspase-1, contributes to macrophage killing and disease progression in LT-treated susceptible rats and mice. Our results further point to a role for inflammatory mediators and cytokines in LT-mediated disease progression. ${ }^{20-22,42}$

\section{Conclusion and Therapeutic Potential of the Study}

Caspase-1 is a pro-inflammatory protein that is involved in activating the innate immune response, and animals lacking functional caspase- 1 are usually impaired in their ability to control a systemic infection by microbial pathogens. ${ }^{43-45}$ Our findings, however, suggest that overstimulation or dysregulation of caspase-1 can also result in disease progression and vascular collapse. It is conceivable that macrophage necrosis results in the release of inflammatory factors that drive disease progression. It remains to be shown whether putative factor(s) released by necrotic cells are responsible for the systemic shock mediated by LT and other NLR inducers.

Our results showed that proteasome inhibitors were able to prevent/slow down both macrophage killing and vascular shock in rodents exposed to LT, presumably by preventing caspase- 1 activation. The ability to delay or prevent LT-mediated disease progression in rodents is a novel application of NPI-0052, bortezomib, and proteasome inhibitors in general. It is possible that greater protection could be obtained with a combination of proteasome inhibitors, which have been shown to exhibit synergistic effects. ${ }^{37}$ A combination therapy might protect at lower inhibitor concentrations with reduced side effects and improved safety profile. ${ }^{37}$

To our knowledge, this is first study to demonstrate that it is possible to treat shock-like-syndromes by pharmaceutical inhibition of caspase-1. Proteasome inhibitors and other drugs targeting the inflammasome/caspase-1 may prove effective in treating NLR-associated inflammatory diseases. Given that many microbial pathogens including Bacillus anthracis trigger a shock-like syndrome ${ }^{46}$ therapeutic approaches that reduce inflammation might improve survival. Our findings indicate the therapeutic potential of drugs targeting the inflammasome for the treatment of inflammatory and microbial diseases.

\section{Acknowledgments}

We thank Raynal Squires for critical reading of the article, and Wang Yong Zeng for technical assistance.

\section{References}

1. Mariathasan S, Newton K, Monack DM, Vucic D, French DM, Lee WP Roose-Girma M, Erickson S, Dixit VM: Differential activation of the inflammasome by caspase-1 adaptors ASC and Ipaf. Nature 2004, 430:213-218

2. Mariathasan S, Weiss DS, Dixit VM, Monack DM: Innate immunity against Francisella tularensis is dependent on the ASC/caspase-1 axis. J Exp Med 2005, 202:1043-1049

3. Mariathasan S, Weiss DS, Newton K, McBride J, O'Rourke K, RooseGirma M, Lee WP, Weinrauch Y, Monack DM, Dixit VM: Cryopyrin activates the inflammasome in response to toxins and ATP. Nature 2006, 440:228-232

4. Boyden ED, Dietrich WF: Nalp1b controls mouse macrophage susceptibility to anthrax lethal toxin. Nat Genet 2006, 38:240-244

5. Fink SL, Bergsbaken T, Cookson BT: Anthrax lethal toxin and Salmonella elicit the common cell death pathway of caspase-1-dependent pyroptosis via distinct mechanisms. Proc Natl Acad Sci USA 2008, 105:4312-4317

6. Muehlbauer SM, Evering TH, Bonuccelli G, Squires RC, Ashton AW Porcelli SA, Lisanti MP, Brojatsch J: Anthrax lethal toxin kills macrophages in a strain-specific manner by apoptosis or caspase-1-mediated necrosis. Cell Cycle 2007, 6:758-766 
7. Wickliffe KE, Leppla SH, Moayeri M: Anthrax lethal toxin-induced inflammasome formation and caspase-1 activation are late events dependent on ion fluxes and the proteasome. Cell Microbiol 2008, 10:332-343

8. Collier RJ, Young JA: Anthrax toxin. Annu Rev Cell Dev Biol 2003, 19:45-70

9. Forino M, Johnson S, Wong TY, Rozanov DV, Savinov AY, Li W Fattorusso R, Becattini B, Orry AJ, Jung D, Abagyan RA, Smith JW, Alibek K, Liddington RC, Strongin AY, Pellecchia M: Efficient synthetic inhibitors of anthrax lethal facto. Proc Natl Acad Sci USA 2005, 102:9499-9504

10. Shoop WL, Xiong Y, Wiltsie J, Woods A, Guo J, Pivnichny JV, Felcetto T, Michael BF, Bansal A, Cummings RT, Cunningham BR, Friedlander AM, Douglas CM, Patel SB, Wisniewski D, Scapin G, Salowe SP, Zaller DM, Chapman KT, Scolnick EM, Schmatz DM, Bartizal K, MacCoss M, Hermes JD: Anthrax lethal factor inhibition. Proc Natl Acad Sci USA 2005, 102:7958-7963

11. Hsu LC, Ali SR, McGillivray S, Tseng PH, Mariathasan S, Humke EW, Eckmann L, Powell JJ, Nizet V, Dixit VM, Karin M: A NOD2-NALP1 complex mediates caspase-1-dependent IL-1beta secretion in response to Bacillus anthracis infection and muramyl dipeptide. Proc Natl Acad Sci USA 2008, 105:7803-7808

12. Alileche A, Serfass ER, Muehlbauer SM, Porcelli SA, Brojatsch J: Anthrax lethal toxin-mediated killing of human and murine dendritic cells impairs the adaptive immune response. PLoS Pathog 2005 , 1:e19

13. Reig N, Jiang A, Couture R, Sutterwala FS, Ogura $Y$, Flavell RA, Mellman I, van der Goot FG: Maturation modulates caspase-1-independent responses of dendritic cells to Anthrax lethal toxin. Cell Microbiol 2008, 10:1190-1207

14. Nour AM, Yeung YG, Santambrogio L, Boyden ED, Stanley ER, Brojatsch J: Anthrax lethal toxin triggers the formation of a membraneassociated inflammasome complex in murine macrophages. Infect Immun 2009, 77:1262-1271

15. Squires RC, Muehlbauer SM, Brojatsch J: Proteasomes control caspase-1 activation in anthrax lethal toxin-mediated cell killing. J Biol Chem 2007, 282:34260-34267

16. Cordoba-Rodriguez R, Fang H, Lankford CS, Frucht DM: Anthrax lethal toxin rapidly activates caspase-1/ICE and induces extracellular release of interleukin (IL)-1beta and IL-18. J Biol Chem 2004, 279:20563-20566

17. Chapelsky S, Batty S, Frost M, Mogridge J: Inhibition of anthrax lethal toxin-induced cytolysis of RAW264.7 cells by celastrol. PLoS One 2008, 3:e1421

18. Bergsbaken T, Cookson BT: Macrophage activation redirects yersinia-infected host cell death from apoptosis to caspase-1-dependent pyroptosis. PLoS Pathog 2007, 3:e161

19. Nye SH, Wittenburg AL, Evans DL, O'Connor JA, Roman RJ, Jacob $\mathrm{HJ}$ : Rat survival to anthrax lethal toxin is likely controlled by a single gene. Pharmacogenomics J 2008, 8:16-22

20. Sherer K, Li Y, Cui X, Li X, Subramanian M, Laird MW, Moayeri M, Leppla SH, Fitz Y, Su J, Eichacker PQ: Fluid support worsens outcome and negates the benefit of protective antigen-directed monoclonal antibody in a lethal toxin-infused rat Bacillus anthracis shock model. Crit Care Med 2007, 35:1560-1567

21. Cui X, Moayeri M, Li Y, Li X, Haley M, Fitz Y, Correa-Araujo R, Banks SM, Leppla SH, Eichacker PQ: Lethality during continuous anthrax lethal toxin infusion is associated with circulatory shock but not inflammatory cytokine or nitric oxide release in rats. Am J Physiol Regul Integr Comp Physiol 2004, 286:R699-R709

22. Kuo SR, Willingham MC, Bour SH, Andreas EA, Park SK, Jackson C, Duesbery NS, Leppla SH, Tang WJ, Frankel AE: Anthrax toxin-induced shock in rats is associated with pulmonary edema and hemorrhage. Microb Pathog 2008, 44:467-472

23. Boltz-Nitulescu G, Wiltschke C, Holzinger C, Fellinger A, Scheiner O, Gessl A, Forster O: Differentiation of rat bone marrow cells into macrophages under the influence of mouse L929 cell supernatant. J Leukoc Biol 1987, 41:83-91

24. Perez-Perez GI, Shepherd VL, Morrow JD, Blaser MJ: Activation of human THP-1 cells and rat bone marrow-derived macrophages by Helicobacter pylori lipopolysaccharide. Infect Immun 1995 63:1183-1187
25. Akoachere M, Squires RC, Nour AM, Angelov L, Brojatsch J, AbelSantos E: Identification of an in vivo inhibitor of Bacillus anthracis spore germination. J Biol Chem 2007, 282:12112-12118

26. Diaz-Griffero F, Hoschander SA, Brojatsch J: Bystander killing during avian leukosis virus subgroup $B$ infection requires TVB(S3) signaling. J Virol 2003, 77:12552-12561

27. Alileche K, Squires RC, Muehlbauer SM, M.P. L, Brojatsch J: Mitochondrial impairment mediates cytolysis in anthrax lethal toxintreated murine macrophages. Cell Cycle 2006, 5:100-106

28. Goldman D, Lee SC, Casadevall A: Pathogenesis of pulmonary Cryptococcus neoformans infection in the rat. Infect Immun 1994, 62:4755-4761

29. Watters JW, Dietrich WF: Genetic, physical, and transcript map of the Ltxs1 region of mouse chromosome 11. Genomics 2001, 73:223-231

30. Moayeri M, Haines D, Young HA, Leppla SH: Bacillus anthracis lethal toxin induces TNF-alpha-independent hypoxia-mediated toxicity in mice. J Clin Invest 2003, 112:670-682

31. Moayeri M, Martinez NW, Wiggins J, Young HA, Leppla SH: Mouse susceptibility to anthrax lethal toxin is influenced by genetic factors in addition to those controlling macrophage sensitivity. Infect Immun 2004, 72:4439-4447

32. Averette KM, Pratt MR, Yang Y, Bassilian S, Whitelegge JP, Loo JA, Muir TW, Bradley KA: Anthrax lethal toxin induced lysosomal membrane permeabilization and cytosolic cathepsin release is NIrp1b/ Nalp1b-dependent. PLoS One 2009, 4:e7913

33. Bergsbaken T, Cookson BT: Innate immune response during Yersinia infection: critical modulation of cell death mechanisms through phagocyte activation. J Leukoc Biol 2009, 86:1153-1158

34. Brough D, Rothwell NJ: Caspase-1-dependent processing of prointerleukin-1beta is cytosolic and precedes cell death. J Cell Sci 2007, 120:772-781

35. Lara-Tejero M, Sutterwala FS, Ogura Y, Grant EP, Bertin J, Coyle AJ, Flavell RA, Galan JE: Role of the caspase-1 inflammasome in Salmonella typhimurium pathogenesis. J Exp Med 2006, 203:1407-1412

36. Tang G, Leppla SH: Proteasome activity is required for anthrax lethal toxin to kill macrophages. Infect Immun 1999, 67:3055-3060

37. Chauhan D, Singh A, Brahmandam M, Podar K, Hideshima T, Richardson P, Munshi N, Palladino MA, Anderson KC: Combination of proteasome inhibitors bortezomib and NPI-0052 trigger in vivo synergistic cytotoxicity in multiple myeloma. Blood 2008, 111:1654-1664

38. Nett M, Gulder TA, Kale AJ, Hughes CC, Moore BS: Function-oriented biosynthesis of beta-lactone proteasome inhibitors in Salinispora tropica. J Med Chem 2009, 52:6163-6167

39. Baritaki S, Chapman A, Yeung K, Spandidos DA, Palladino M Bonavida B: Inhibition of epithelial to mesenchymal transition in metastatic prostate cancer cells by the novel proteasome inhibitor. NPI0052: pivotal roles of Snail repression and RKIP induction. Oncogene 2009, 28:3573-3585

40. Manam RR, McArthur KA, Chao TH, Weiss J, Ali JA, Palombella VJ, Groll M, Lloyd GK, Palladino MA, Neuteboom ST, Macherla VR, Potts $\mathrm{BC}$ : Leaving groups prolong the duration of $20 \mathrm{~S}$ proteasome inhibition and enhance the potency of salinosporamides. J Med Chem 2008, 51:6711-6724

41. Elshal MF, McCoy JP: Multiplex bead array assays: performance evaluation and comparison of sensitivity to ELISA. Methods 2006, 38:317-323

42. Watson LE, Kuo SR, Katki K, Dang T, Park SK, Dostal DE, Tang WJ, Leppla SH, Frankel AE: Anthrax toxins induce shock in rats by depressed cardiac ventricular function. PLoS One 2007, 2:e466

43. Kang TJ, Basu S, Zhang L, Thomas KE, Vogel SN, Baillie L, Cross AS: Bacillus anthracis spores and lethal toxin induce IL-1beta via functionally distinct signaling pathways. Eur J Immunol 2008, 38:1574-1584

44. Raupach B, Peuschel SK, Monack DM, Zychlinsky A: Caspase-1mediated activation of interleukin-1beta (IL-1beta) and IL-18 contributes to innate immune defenses against Salmonella enterica serovar Typhimurium infection. Infect Immun 2006, 74:4922-4926

45. Weiss DS, Henry T, Monack DM: Francisella tularensis: activation of the inflammasome. Ann NY Acad Sci 2007, 1105:219-237

46. Goldman DL, Casadevall A: Anthrax-associated shock. Front Biosci 2008, 13:4009-4014 